\title{
Minimal Solution of Complex Fuzzy Linear Systems
}

\author{
Xiaobin Guo and Ke Zhang \\ College of Mathematics and Statistics, Northwest Normal University, Lanzhou 730070, China \\ Correspondence should be addressed to Xiaobin Guo; guoxb@nwnu.edu.cn
}

Received 19 August 2016; Revised 9 November 2016; Accepted 14 November 2016

Academic Editor: Rahib H. Abiyev

Copyright (c) $2016 \mathrm{X}$. Guo and K. Zhang. This is an open access article distributed under the Creative Commons Attribution License, which permits unrestricted use, distribution, and reproduction in any medium, provided the original work is properly cited.

This paper investigates the complex fuzzy linear equation $C \widetilde{z}=\widetilde{w}$ in which $C$ is a crisp complex matrix and $\widetilde{w}$ is an arbitrary LR complex fuzzy vector. The complex fuzzy linear system is converted to equivalent high order fuzzy linear system $G \tilde{x}=\widetilde{b}$. A new numerical procedure for calculating the complex fuzzy solution is designed and a sufficient condition for the existence of strong complex fuzzy solution is derived in detail. Some examples are given to illustrate the proposed method.

\section{Introduction}

In the mathematical modeling of physics, engineering computation, and statistical analysis, it is the linear systems that have mature theory and easy computational property. However, the uncertainty of the parameters is involved in the process of actual mathematical modeling, which is often represented by fuzzy numbers. So the investigation of theory and computing method for fuzzy linear systems plays an important role in the fuzzy mathematics and its applications. The concept of fuzzy numbers and arithmetic operations with these numbers were firstly introduced and investigated by Zadeh [1], Dubois and Prade [2], and Nahmias [3]. A different approach to fuzzy numbers and the structure of fuzzy number spaces were given by Puri and Ralescu [4], Goetschell and Voxman [5], and Wu and Ma [6, 7].

Since Friedman et al. [8] proposed a general model for solving fuzzy linear systems by an embedding approach, in the past decades, a lot of researches about some advanced fuzzy linear systems such as dual fuzzy linear systems (DFLS), general fuzzy linear systems (GFLS), full fuzzy linear systems (FFLS), dual full fuzzy linear systems (DFFLS), and general dual fuzzy linear systems (GDFLS) have emerged. See [920]. In general, the uncertain elements of fuzzy linear systems were denoted by the parametric form of fuzzy numbers and the systems were extended into crisp function linear systems.
Thus, it may lead to two defects. The one is that the extended linear equations always contain parameter $r, 0 \leq r \leq$ 1 , which makes their computation inconvenient in some sense. The other is that the weak fuzzy solution of fuzzy linear systems does not exist [21] sometimes. To make the multiplication of fuzzy numbers easy, Dubois and Prade [2] introduced the LR fuzzy number. We know that triangular fuzzy numbers are just specious cases of LR fuzzy numbers. In 2006, Dehghan et al. [18] discussed computational methods for fully fuzzy linear systems $\widetilde{A} \widetilde{x}=\widetilde{b}$ whose coefficient matrix and the right-hand side vector are LR fuzzy numbers. In the past decade, some researchers paid more attention to LR fuzzy linear systems. In 2013, Guo and Shang [22] proposed a computing method for the fuzzy Sylvester matrix equations $A \widetilde{X}+\widetilde{X} B=\widetilde{C}$ with LR fuzzy numbers. Later, Gong et al. [23] studied the general dual fuzzy linear matrix systems $A \widetilde{X}+\widetilde{B}=$ $C \widetilde{X}+\widetilde{D}$ based on LR fuzzy numbers.

For complex fuzzy system of linear equations, few researchers have developed methods to solve them. The fuzzy complex numbers were introduced firstly by Buckley [24] in 1989. In 2010, Jahantigh et al. [25] studied firstly the $n \times n$ fuzzy complex linear systems. Solution of fuzzy complex linear system of linear equations was described and was applied to circuit analysis problem by Rahgooy et al. [26]. In 2014, Behera and Chakraverty [27] discussed the fuzzy complex 
system of linear equations by the embedding method and redefined the complex fuzzy number [28]. In this paper the LR complex fuzzy linear system $C \widetilde{z}=\widetilde{w}$ is investigated. A numerical procedure for calculating the fuzzy solution is designed and a sufficient condition for the existence of strong fuzzy solution is derived. Finally, some examples are given to illustrate our method.

\section{Preliminaries}

There are some basic definitions and results for fuzzy numbers.

\subsection{The Fuzzy Number}

Definition 1 (see [1]). A fuzzy number is a fuzzy set like $u$ : $R \rightarrow I=[0,1]$ which satisfies the following:

(1) $u$ is upper semicontinuous.

(2) $u$ is fuzzy convex: that is, $u(\lambda x+(1-\lambda) y) \geq$ $\min \{u(x), u(y)\}$ for all $x, y \in R, \lambda \in[0,1]$.

(3) $u$ is normal: that is, there exists $x_{0} \in R$ such that $u\left(x_{0}\right)=1$.

(4) supp $u=\{x \in R \mid u(x)>0\}$ is the support of $u$, and its closure $\mathrm{cl}(\operatorname{supp} u)$ is compact.

Let $E^{1}$ be the set of all fuzzy numbers on $R$.

Definition 2 (see [2]). A fuzzy number $\widetilde{M}$ is said to be a LR fuzzy number if

$$
\mu_{\widetilde{M}}(x)= \begin{cases}L\left(\frac{m-x}{\alpha}\right), & x \leq m, \alpha>0, \\ R\left(\frac{x-m}{\beta}\right), & x \geq m, \beta>0,\end{cases}
$$

where $m, \alpha$, and $\beta$ are called the mean value and left and right spreads of $\widetilde{M}$, respectively. The function $L(\cdot)$, which is called left shape function, satisfies the following:

(1) $L(x)=L(-x)$.

(2) $L(0)=1$ and $L(1)=0$.

(3) $L(x)$ is nonincreasing on $[0, \infty)$.

The definition of a right shape function $R(\cdot)$ is similar to that of $L(\cdot)$.

Clearly, two LR fuzzy numbers $\widetilde{M}=(m, \alpha, \beta)_{\mathrm{LR}}$ and $\widetilde{N}=$ $(n, \gamma, \delta)_{\mathrm{LR}}$ are said to be equal, if and only if $m=n, \alpha=\gamma$ and $\beta=\delta$. Also, $\widetilde{M}=(m, \alpha, \beta)_{\mathrm{LR}}$ is positive (negative) if and only if $m-\alpha>0(m+\beta<0)$.

Definition 3 (see [2]). For arbitrary LR fuzzy numbers $\widetilde{M}=$ $(m, \alpha, \beta)_{\mathrm{LR}}$ and $\widetilde{N}=(n, \gamma, \delta)_{\mathrm{LR}}$, we have the following:

(1) Addition:

$$
\begin{aligned}
\widetilde{M} \oplus \widetilde{N} & =(m, \alpha, \beta)_{\mathrm{LR}} \oplus(n, \gamma, \delta)_{\mathrm{LR}} \\
& =(m+n, \alpha+\gamma, \beta+\delta)_{\mathrm{LR}} .
\end{aligned}
$$

(2) Subtraction:

$$
\begin{aligned}
\widetilde{M}-\widetilde{N} & =(m, \alpha, \beta)_{\mathrm{LR}}-(n, \gamma, \delta)_{\mathrm{LR}} \\
& =(m-n, \alpha-\delta, \beta-\gamma)_{\mathrm{LR}} .
\end{aligned}
$$

(3) Scalar multiplication:

$$
\begin{aligned}
\lambda \otimes \widetilde{M} & =\lambda \otimes(m, \alpha, \beta)_{\mathrm{LR}} \\
& \cong \begin{cases}(\lambda m, \lambda \alpha, \lambda \beta)_{\mathrm{LR}}, & \lambda \geq 0, \\
(\lambda m,-\lambda \beta,-\lambda \alpha)_{\mathrm{RL}}, & \lambda<0 .\end{cases}
\end{aligned}
$$

Definition 4. An arbitrary LR complex fuzzy number should be represented as $\widetilde{x}=\widetilde{p}+i \widetilde{q}$, where $\widetilde{p}=\left(p, p^{l}, p^{r}\right)$ and $\widetilde{q}=\left(q, q^{l}, q^{r}\right)$. Likely, $\widetilde{x}$ can be written as $\tilde{x}=\left(p, p^{l}, p^{r}\right)+$ $i\left(q, q^{l}, q^{r}\right)$.

Definition 5. For any two arbitrary complex fuzzy numbers $\widetilde{x}=\widetilde{p}+i \widetilde{q}$ and $\tilde{y}=\widetilde{u}+i \widetilde{v}$ where $\widetilde{p}, \widetilde{q}, \widetilde{u}, \widetilde{v}$ are fuzzy numbers, their arithmetic is as follows:
(1) $\tilde{x}+\tilde{y}=(\widetilde{p}+\widetilde{u})+i(\widetilde{q}+\widetilde{v})$,
(2) $k \tilde{x}=k \tilde{p}+i k \tilde{q}, k \in R$,
(3) $\tilde{x} \times \tilde{y}=(\tilde{p} \times \tilde{u}-\widetilde{q} \times \widetilde{v})+i(\widetilde{p} \times \widetilde{v}+\widetilde{q} \times \widetilde{u})$.

\subsection{Complex Fuzzy Linear Systems}

Definition 6. The linear system equation

$$
\left(\begin{array}{cccc}
c_{11} & c_{12} & \cdots & c_{1 n} \\
c_{21} & c_{12} & \cdots & c_{2 n} \\
\cdots & \cdots & \cdots & \cdots \\
c_{n 1} & c_{n 2} & \cdots & c_{n n}
\end{array}\right)\left(\begin{array}{c}
\widetilde{z}_{1} \\
\widetilde{z}_{2} \\
\vdots \\
\widetilde{z}_{n}
\end{array}\right)=\left(\begin{array}{c}
\widetilde{w}_{1} \\
\widetilde{w}_{2} \\
\vdots \\
\widetilde{w}_{n}
\end{array}\right)
$$

where $c_{i j}, 1 \leq i, j \leq n$ are LR complex numbers and $\widetilde{w}_{i}, 1 \leq$ $i \leq n$ are complex fuzzy numbers, is called a LR complex fuzzy linear system (CFLS).

Using matrix notation, we have

$$
C \widetilde{z}=\widetilde{w} .
$$

A complex fuzzy numbers vector

$$
\widetilde{z}=\left(\widetilde{z}_{1}, \widetilde{z}_{2}, \ldots, \widetilde{z}_{n}\right)^{\top}
$$

is called a fuzzy solution of the complex fuzzy linear system (5) if $\widetilde{z}$ satisfies (6).

\section{Solving Complex Fuzzy Linear System}

Definition 7. For two LR fuzzy numbers vectors $\tilde{x}=\left(x, x^{l}, x^{r}\right)$ and $\tilde{y}=\left(y, y^{l}, y^{r}\right)$, we have the following:

(1) Addition:

$$
\begin{aligned}
\tilde{x}+\tilde{y} & =\left(x, x^{l}, x^{r}\right)+\left(y, y^{l}, y^{r}\right) \\
& =\left(x+y, x^{l}+y^{l}, x^{r}+y^{r}\right) .
\end{aligned}
$$


(2) Subtraction:

$$
\begin{aligned}
\tilde{x}-\tilde{y} & =\left(x, x^{l}, x^{r}\right)-\left(y, y^{l}, y^{r}\right) \\
& =\left(x+y, x^{l}-y^{r}, x^{r}-y^{l}\right) .
\end{aligned}
$$

(3) Scalar multiplication:

$$
\lambda \widetilde{x}=\lambda\left(x, x^{l}, x^{r}\right) \cong \begin{cases}\left(\lambda x, \lambda x^{l}, \lambda x^{r}\right), & \lambda \geq 0, \\ \left(\lambda x,-\lambda x^{r},-\lambda x^{l}\right), & \lambda<0 .\end{cases}
$$

Definition 8. An arbitrary LR complex fuzzy vector should be represented as $\widetilde{x}=\widetilde{p}+i \widetilde{q}$, where $\widetilde{p}=\left(p, p^{l}, p^{r}\right)$ and $\widetilde{q}=$ $\left(q, q^{l}, q^{r}\right)$ are two LR fuzzy numbers vectors. Likely, the LR complex fuzzy vector $\tilde{x}$ can be written as

$$
\tilde{x}=\left(p, p^{l}, p^{r}\right)+i\left(q, q^{l}, q^{r}\right) \text {. }
$$

\subsection{Equivalent Fuzzy Linear System}

Theorem 9. The $n \times n$ complex fuzzy linear system (5) is equivalent to $2 n \times 2 n$ order fuzzy linear system:

$$
G \tilde{x}=\tilde{b},
$$

where

$$
\begin{aligned}
G & =\left(\begin{array}{cc}
A & -B \\
B & A
\end{array}\right), \\
\tilde{x} & =\left(\begin{array}{l}
\widetilde{p} \\
\tilde{q}
\end{array}\right), \\
\widetilde{b} & =\left(\begin{array}{l}
\widetilde{u} \\
\widetilde{v}
\end{array}\right) .
\end{aligned}
$$

Proof. We denote $C=A+i B, A, B \in R^{n \times n}$ and $\widetilde{w}=\tilde{u}+i \widetilde{v}$, where $\tilde{u}$ and $\widetilde{v}$ are fuzzy number vectors. We also suppose the unknown vector $\widetilde{z}=\widetilde{p}+i \widetilde{q}$, where $\widetilde{p}$ and $\tilde{q}$ are two unknown fuzzy number vectors.

Since $C \widetilde{z}=\widetilde{w}$, we have

$$
(A+i B)(\widetilde{p}+i \widetilde{q})=\widetilde{u}+i \widetilde{v} .
$$

That is,

$$
(A \widetilde{p}-B \widetilde{q})+i(A \widetilde{q}+B \widetilde{p})=\widetilde{u}+i \widetilde{v} .
$$

Comparing with the coefficient of $i$, we have

$$
\begin{aligned}
& A \widetilde{p}-B \widetilde{q}=\widetilde{u}, \\
& A \widetilde{q}+B \widetilde{p}=\widetilde{v} .
\end{aligned}
$$

That is,

$$
\left(\begin{array}{cc}
A & -B \\
B & A
\end{array}\right)\left(\begin{array}{l}
\tilde{p} \\
\tilde{q}
\end{array}\right)=\left(\begin{array}{l}
\tilde{u} \\
\widetilde{v}
\end{array}\right),
$$

which admits $2 n$ order fuzzy linear system.

We express it in matrix form as follows:

$$
G \tilde{x}=\tilde{b} .
$$

3.2. Solving CFLS. In order to solve the complex fuzzy linear system (5), we need to solve the real fuzzy system of linear equations (12).

Firstly, we set up a computing model for solving LR CFLS. Then we define the complex fuzzy solution of CFLS and obtain its solution representation by the generalized inverses of matrices.

According to operations of LR fuzzy numbers, we have the following results.

Theorem 10. The fuzzy linear system (12) can be extended into the following system of linear matrix equations:

$$
\begin{gathered}
\left(G^{+}+G^{-}\right) x=b \\
\left(\begin{array}{cc}
G^{+} & -G^{-} \\
-G^{-} & G^{+}
\end{array}\right)\left(\begin{array}{l}
x^{l} \\
x^{r}
\end{array}\right)=\left(\begin{array}{c}
b^{l} \\
b^{r}
\end{array}\right),
\end{gathered}
$$

where

$$
\begin{aligned}
& G=\left(\begin{array}{cc}
A & -B \\
B & A
\end{array}\right)=G^{+}+G^{-}, \\
& \tilde{x}=\left(x, x^{l}, x^{r}\right)=\left(\left(\begin{array}{l}
p \\
q
\end{array}\right)\left(\begin{array}{l}
p^{l} \\
q^{l}
\end{array}\right)\left(\begin{array}{l}
p^{r} \\
q^{r}
\end{array}\right)\right), \\
& \tilde{b}=\left(b, b^{l}, b^{r}\right)=\left(\left(\begin{array}{l}
u \\
v
\end{array}\right)\left(\begin{array}{l}
u^{l} \\
v^{l}
\end{array}\right)\left(\begin{array}{l}
u^{r} \\
v^{r}
\end{array}\right)\right),
\end{aligned}
$$

where the elements $g_{i j}^{+}$and $g_{i j}^{-}$are determined as follows: if $g_{i j} \geq$ $0, g_{i j}^{+}=g_{i j}$ then $g_{i j}^{+}=0,1 \leq i, j \leq 2 n$; if $g_{i j}<0, g_{i j}^{-}=g_{i j}$ then $g_{i j}^{-}=0,1 \leq i, j \leq 2 n$.

Proof. Denoting $\widetilde{z}=\left(p, p^{l}, p^{r}\right)+i\left(q, q^{l}, q^{r}\right), \widetilde{w}=\left(u, u^{l}, u^{r}\right)+$ $i\left(v, v^{l}, v^{r}\right)$, where $p, q$ are the center values and $p^{l}, p^{r}, q^{l}, q^{r}$ are the left and right spread values of fuzzy number vectors $\widetilde{p}, \widetilde{q}$, respectively, then the fuzzy linear system $G \tilde{x}=\widetilde{b}$ is

$$
G\left(x, x^{l}, x^{r}\right)=\left(b, b^{l}, b^{r}\right) .
$$

Let

$$
G=\left(\begin{array}{cc}
A & -B \\
B & A
\end{array}\right)=G^{+}+G^{-},
$$

where the elements $g_{i j}^{+}$and $g_{i j}^{-}$are determined as follows: if $g_{i j} \geq 0, g_{i j}^{+}=g_{i j}$ then $g_{i j}^{+}=0,1 \leq i, j \leq 2 n$; if $g_{i j}<0, g_{i j}^{-}=$ $g_{i j}$ then $g_{i j}^{-}=0,1 \leq i, j \leq 2 n$.

Since

$$
k \tilde{x}_{j}= \begin{cases}\left(k x_{j}, k x_{j}^{l}, k x_{j}^{r}\right), & k \geq 0, \\ \left(k x_{j},-k x_{j}^{r},-k x_{j}^{l}\right), & k<0,\end{cases}
$$

we have

$$
G \widetilde{x}= \begin{cases}\left(G x, G x^{l}, G x^{r}\right), & G \geq 0, \\ \left(G x,-G x^{r},-G x^{l}\right), & G<0 .\end{cases}
$$


The equation

$$
\left(G^{+}+G^{-}\right)\left(x, x^{l}, x^{r}\right)=\left(b, b^{l}, b^{r}\right)
$$

is equivalent to

$$
\begin{aligned}
G^{+} & \left(x, x^{l}, x^{r}\right)+G^{-}\left(x, x^{l}, x^{r}\right) \\
& =\left(G^{+} x, G^{+} x^{l}, G^{+} x^{r}\right)+\left(G^{-} x,-G^{-} x^{r},-G^{-} x^{l}\right) \\
& =\left(G^{+} x+G^{-} x, G^{+} x^{l}-G^{-} x^{r}, G^{+} x^{r}-G^{-} x^{l}\right) \\
& =\left(b, b^{l}, b^{r}\right) .
\end{aligned}
$$

Thus, we have

$$
\begin{aligned}
& \left(G^{+}+G^{-}\right) x=b, \\
& G^{+} x^{l}-G^{-} x^{r}=b^{l}, \\
& G^{+} x^{r}-G^{-} x^{l}=b^{r} .
\end{aligned}
$$

Denoting (27) in a matrix form, we have

$$
\begin{aligned}
& \left(G^{+}+G^{-}\right)\left(\begin{array}{l}
p \\
q
\end{array}\right)=\left(\begin{array}{l}
u \\
v
\end{array}\right), \\
& \left(\begin{array}{cc}
G^{+} & -G^{-} \\
-G^{-} & G^{+}
\end{array}\right)\left(\begin{array}{c}
p^{l} \\
q^{l} \\
p^{r} \\
q^{r}
\end{array}\right)=\left(\begin{array}{c}
u^{l} \\
v^{l} \\
u^{r} \\
v^{r}
\end{array}\right) .
\end{aligned}
$$

By means of calculations, we obtain the minimal solution of the model equation (19) as follows:

$$
\begin{aligned}
x & =\left(\begin{array}{l}
p \\
q
\end{array}\right)=\left(\begin{array}{cc}
A & -B \\
B & A
\end{array}\right)^{\dagger}\left(\begin{array}{l}
u \\
v
\end{array}\right), \\
\left(\begin{array}{l}
x^{l} \\
x^{r}
\end{array}\right) & =\left(\begin{array}{c}
p^{l} \\
q^{l} \\
p^{r} \\
q^{r}
\end{array}\right)=\left(\begin{array}{cc}
G^{+} & -G^{-} \\
-G^{-} & G^{+}
\end{array}\right)^{\dagger}\left(\begin{array}{c}
u^{l} \\
v^{l} \\
u^{r} \\
v^{r}
\end{array}\right) .
\end{aligned}
$$

It seems that we obtained the fuzzy vector $\tilde{x}$ as the above (29). However, the solution vector $\tilde{x}=\left(x, x^{l}, x^{r}\right)$ may not be an appropriate LR fuzzy number one except for $x^{l} \geq O, x^{r} \geq$ $O$. So we give the definition of LR complex fuzzy solution to complex fuzzy linear system (6) as follows.

Definition 11. Let

$$
\tilde{x}=\left(x, x^{l}, x^{r}\right)=\left(\left(\begin{array}{l}
p \\
q
\end{array}\right)\left(\begin{array}{l}
p^{l} \\
q^{l}
\end{array}\right)\left(\begin{array}{l}
p^{r} \\
q^{r}
\end{array}\right)\right) .
$$

If $\left(x, x^{l}, x^{r}\right)$ is the minimal solution of (19) such that $x^{l} \geq$ $0, x^{r} \geq 0$, we call $\tilde{x}=\left(x, x^{l}, x^{r}\right)_{\mathrm{LR}}$ a strong LR fuzzy minimal solution of (17). Meanwhile, the complex fuzzy vector $\tilde{x}=$ $\left(p, p^{l}, p^{r}\right)+i\left(q, q^{l}, q^{r}\right)$ is called a strong LR complex fuzzy minimal solution of (5). Otherwise, $\tilde{x}=\left(p, p^{l}, p^{r}\right)+i\left(q, q^{l}, q^{r}\right)$ is said to be a weak LR complex fuzzy minimal solution of (5) given by

$$
\tilde{x}_{j}= \begin{cases}\left(x_{j}, x_{j}^{l}, x_{j}^{r}\right)_{\mathrm{LR}}, & x_{j}^{l}>0, x_{j}^{r}>0, \\ \left(x_{j}, 0, \max \left\{-x_{j}^{l}, x_{j}^{r}\right\}\right)_{\mathrm{LR}}, & x_{j}^{l}<0, x_{j}^{r}>0, \\ \left(x_{j}, \max \left\{x_{j}^{l},-x_{j}^{r}\right\}, 0\right)_{\mathrm{LR}}, & x_{j}^{l}>0, x_{j}^{r}<0, \\ \left(x_{j},-x_{j}^{r},-x_{j}^{l}\right)_{\mathrm{LR}}, & x_{j}^{l}<0, x_{j}^{r}<0, \\ & j=1, \ldots, 2 n .\end{cases}
$$

3.3. A Sufficient Condition for Strong Fuzzy Solution. Now we give a sufficient condition for strong fuzzy approximate solution to the complex fuzzy linear system by the following analysis.

To illustrate expression (29) to be a LR fuzzy solution vector, we now discuss the generalized inverses of nonnegative matrix

$$
S=\left(\begin{array}{cc}
G^{+} & -G^{-} \\
-G^{-} & G^{+}
\end{array}\right)
$$

in a special structure.

Lemma 12 (see [29]). Let

$$
S=\left(\begin{array}{cc}
G^{+} & -G^{-} \\
-G^{-} & G^{+}
\end{array}\right) \text {. }
$$

Then the matrix

$$
\begin{aligned}
& S^{\dagger}=\frac{1}{2} \\
& \left(\begin{array}{cc}
\left(G^{+}-G^{-}\right)^{\dagger}+\left(G^{+}+G^{-}\right)^{\dagger} & \left(G^{+}-G^{-}\right)^{\dagger}-\left(G^{+}+G^{-}\right)^{\dagger} \\
\left(G^{+}-G^{-}\right)^{\dagger}-\left(G^{+}+G^{-}\right)^{\dagger} & \left(G^{+}-G^{-}\right)^{\dagger}+\left(G^{+}+G^{-}\right)^{\dagger}
\end{array}\right)
\end{aligned}
$$

is the Moore-Penrose inverse of the matrix $S$, where $\left(G^{+}+\right.$ $\left.A^{-}\right)^{\dagger},\left(G^{+}-G^{-}\right)^{\dagger}$ are Moore-Penrose inverses of matrices $G^{+}+$ $G^{-}$and $G^{+}-G^{-}$, respectively.

The key point to make the solution vector be a strong LR fuzzy solution is that $\widetilde{x}=\left(x, x^{l}, x^{r}\right)$ is LR fuzzy vector, in which each element is a LR fuzzy number. By the following analysis, we know that it is equivalent to the condition $S^{\dagger} \geq O$.

Theorem 13. If

$$
\begin{aligned}
& \left(G^{+}-G^{-}\right)^{\dagger}+\left(G^{+}+G^{-}\right)^{\dagger} \geq O, \\
& \left(G^{+}-G^{-}\right)^{\dagger}-\left(G^{+}+G^{-}\right)^{\dagger} \geq O,
\end{aligned}
$$

the fuzzy linear equation (5) has a strong LR complex fuzzy minimal solution as follows:

$$
\tilde{x}=\left(p, p^{l}, p^{r}\right)+i\left(q, q^{l}, q^{r}\right),
$$


where

$$
\begin{gathered}
x=G^{\dagger} b \\
\left(\begin{array}{l}
\left(\begin{array}{l}
p^{l} \\
q^{l}
\end{array}\right) \\
\left(\begin{array}{l}
p^{r} \\
q^{r}
\end{array}\right)
\end{array}\right)=\left(\begin{array}{c}
x^{l} \\
x^{r}
\end{array}\right)=\left(\begin{array}{cc}
G^{+} & -G^{-} \\
-G^{-} & G^{+}
\end{array}\right)^{\dagger}\left(\begin{array}{l}
b^{l} \\
b^{r}
\end{array}\right) .
\end{gathered}
$$

Further, if $G^{\dagger} b-E b^{l}-F b^{R} \geq 0$, the fuzzy linear equation (5) has a nonnegative strong $L R$ complex fuzzy minimal solution.

Proof. Since $b^{l}$ and $b^{r}$ are the left and right spreads fuzzy matrix $\widetilde{b}, b^{l} \geq O$ and $b^{r} \geq O$. It means $\left(b^{l}, b^{r}\right)$ is a nonnegative matrix.

Let

$$
\begin{aligned}
S^{\dagger} & =\left(\begin{array}{ll}
E & F \\
F & E
\end{array}\right)=\frac{1}{2} \\
& \cdot\left(\begin{array}{l}
\left(G^{+}-G^{-}\right)^{\dagger}+\left(G^{+}+G^{-}\right)^{\dagger}\left(G^{+}-G^{-}\right)^{\dagger}-\left(G^{+}+G^{-}\right)^{\dagger} \\
\left(G^{+}-G^{-}\right)^{\dagger}-\left(G^{+}+G^{-}\right)^{\dagger}\left(G^{+}-G^{-}\right)^{\dagger}+\left(G^{+}+G^{-}\right)^{\dagger}
\end{array}\right) .
\end{aligned}
$$

We know the condition where $S^{\dagger} \geq 0$ is equivalent to the fact that $E \geq 0$ and $F \geq 0$.

Now that $E \geq O$ and $F \geq O$, the product of two nonnegative matrices

$$
\left(\begin{array}{c}
x^{l} \\
x^{r}
\end{array}\right)=\left(\begin{array}{ll}
E & F \\
F & E
\end{array}\right)\left(\begin{array}{l}
b^{l} \\
b^{r}
\end{array}\right)=\left(\begin{array}{c}
E b^{l}+F b^{r} \\
F b^{l}+E b^{r}
\end{array}\right) \geq O
$$

is nonnegative in nature: that is, $\left(\begin{array}{c}p^{1} \\ q^{l}\end{array}\right)=x^{l} \geq O$ and $\left(\begin{array}{c}p^{r} \\ q^{r}\end{array}\right)=$ $x^{r} \geq O$.

Since $G_{\dagger} b-E b^{l}-F b^{R} \geq 0$, it means that $x-x^{l}=$ $\left(\begin{array}{c}p-p^{l} \\ q-q^{l}\end{array}\right) \geq O$. Thus, we have that the fuzzy linear equation (5) has a nonnegative strong LR complex fuzzy minimal solution by Definition 8 .

The following theorems give some results for such $S^{-1}$ and $S^{\dagger}$ to be nonnegative. As usual, $(\cdot)^{\top}$ denotes the transpose of a matrix $(\cdot)$.

Theorem 14 (see [30]). The inverse $S^{-1}$ of a nonnegative matrix $S$ is nonnegative if and only if $S$ is a generalized permutation matrix.

Theorem 15 (see [31]). Let $S$ be an $2 n \times 2 m$ nonnegative matrix with rank $r$. Then the following assertions are equivalent:

(a) $S^{\dagger} \geq 0$. (b) There exists a permutation matrix $P$, such that PS has the form

$$
P S=\left(\begin{array}{c}
T_{1} \\
T_{2} \\
\vdots \\
T_{r} \\
O
\end{array}\right) \text {, }
$$

where each $T_{i}$ has rank 1 and the rows of $T_{i}$ are orthogonal to the rows of $T_{j}$; whenever $i \neq j$, the zero matrix may be absent.

(c) $S^{\dagger}=\left(\begin{array}{c}G C^{\top} G D^{\top} \\ G D^{\top} G C^{\top}\end{array}\right)$ for some positive diagonal matrix $G$. In this case,

$$
\begin{aligned}
& (C+D)^{\dagger}=G(C+D)^{\top}, \\
& (C-D)^{\dagger}=G(C-D)^{\top} .
\end{aligned}
$$

\section{Numerical Examples}

In this section, we will demonstrate the efficiency of the proposed method by two numerical examples.

Example 1. Consider the following CFSLE:

$$
\begin{aligned}
& \left(\begin{array}{cc}
1 & -1 \\
1 & 3
\end{array}\right)\left(\begin{array}{l}
\widetilde{z}_{1} \\
\tilde{z}_{2}
\end{array}\right) \\
& =\left(\begin{array}{c}
(r, 2-r)+i(1+r, 3-r) \\
(4+r, 7-2 r)+i(r-4,-1-2 r)
\end{array}\right) .
\end{aligned}
$$

Let $\widetilde{z}_{1}=\widetilde{p}_{1}+i \widetilde{q}_{1}=\left(\underline{p_{1}}, \overline{p_{1}}\right)+i\left(\underline{q_{1}}, \overline{q_{1}}\right), \widetilde{z}_{2}=\widetilde{p}_{2}+i \widetilde{q}_{2}=$ $\left(p_{2}, \overline{p_{2}}\right)+i\left(q_{2}, \overline{q_{2}}\right)$. Applying Theorem 9 ., the complex fuzzy linear is equivalent to the following fuzzy linear system $G x=$ $b$ :

$$
\begin{gathered}
\left(\begin{array}{cccc}
1 & -1 & 0 & 0 \\
1 & 3 & 0 & 0 \\
0 & 0 & 1 & -1 \\
0 & 0 & 1 & 3
\end{array}\right)\left(\begin{array}{c}
\tilde{p}_{1} \\
\tilde{p}_{2} \\
\tilde{q}_{1} \\
\tilde{q}_{2}
\end{array}\right) \\
=\left(\begin{array}{c}
(r, 2-r) \\
(4+r, 7-2 r) \\
(1+r, 3-r) \\
(r-4,-1-2 r)
\end{array}\right) .
\end{gathered}
$$


From Theorem 10, we need to solve the following function linear system:

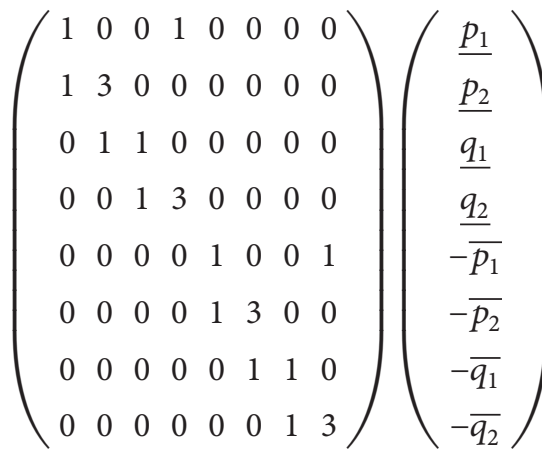

$$
=\left(\begin{array}{c}
r \\
4+r \\
1+r \\
r-4 \\
r-2 \\
2 r-7 \\
r-3 \\
2 r+1
\end{array}\right) .
$$

By (34), we have

$$
\begin{gathered}
\left(\begin{array}{c}
\frac{p_{1}}{p_{2}} \\
\frac{q_{1}}{q_{2}} \\
\frac{\underline{p_{1}}}{-\overline{p_{2}}} \\
-\overline{q_{1}} \\
-\overline{q_{2}}
\end{array}\right)=\left(\begin{array}{cc}
G^{+} & -G^{-} \\
-G^{-} & G^{+}
\end{array}\right)\left(\begin{array}{c}
r \\
4+r \\
1+r \\
r-4 \\
r-2 \\
2 r-7 \\
r-3 \\
2 r+1
\end{array}\right) \\
-\left(\begin{array}{c}
1.375+0.625 r \\
0.875+0.125 r \\
0.125+0.625 r \\
-1.375+0.125 r \\
-2.875+0.875 r \\
-1.375+0.375 r \\
-1.625+0.875 r \\
0.875+0.375 r
\end{array}\right)
\end{gathered}
$$

Thus, we obtain the solution of the complex fuzzy linear system as

$$
\widetilde{z}=\left(\begin{array}{l}
\widetilde{z}_{1} \\
\widetilde{z}_{2}
\end{array}\right)=\left(\begin{array}{c}
(1.375+0.625 r, 2.875-0.875 r)+i(0.125+0.625 r, 1.625-0.875 r) \\
(0.875+0.125 r, 1.375-0.375 r)+i(-1.375+0.125 r,-0.875-0.375 r)
\end{array}\right) .
$$

Example 2. Consider a simple RLC circuit with fuzzy current and fuzzy source; see Figure 1. The CFSLE for the circuit is as follows:

$$
\begin{gathered}
\left(\begin{array}{cc}
10-7.5 i & -6+5 i \\
-6+5 i & 16+3 i
\end{array}\right)\left(\begin{array}{c}
\widetilde{z}_{1} \\
\widetilde{z}_{2}
\end{array}\right) \\
=\left(\begin{array}{c}
(5,1,1)_{\mathrm{LR}}+i(0,1,1)_{\mathrm{LR}} \\
(-1,1,1)_{\mathrm{LR}}+i(-2,1,1)_{\mathrm{LR}}
\end{array}\right) .
\end{gathered}
$$

Let $\widetilde{z}_{1}=\left(p_{1}, p_{1}^{l}, p_{1}^{r}\right)_{\mathrm{LR}}+i\left(q_{1}, q_{1}^{l}, q_{1}^{r}\right)_{\mathrm{LR}}, \widetilde{z}_{2}=\left(p_{2}, p_{2}^{l}\right.$, $\left.p_{2}^{r}\right)_{\mathrm{LR}}+i\left(q_{2}, q_{2}^{l}, q_{2}^{r}\right)_{\mathrm{LR}}$.

According to Theorem 9., the LR complex fuzzy linear is equivalent to the following fuzzy linear system $G x=b$ :

$$
\left(\begin{array}{cccc}
10 & -6 & 7.5 & -5 \\
-6 & 16 & -5 & -3 \\
-7.5 & 5 & 10 & -6 \\
5 & 3 & -6 & 16
\end{array}\right)\left(\begin{array}{l}
\widetilde{p}_{1} \\
\widetilde{p}_{2} \\
\widetilde{q}_{1} \\
\tilde{q}_{2}
\end{array}\right)
$$

$$
=\left(\begin{array}{c}
(5,1,1)_{\mathrm{LR}} \\
(-1,1,1)_{\mathrm{LR}} \\
(0,1,1)_{\mathrm{LR}} \\
(-2,1,1)_{\mathrm{LR}}
\end{array}\right) .
$$

Applying Theorem 10, we need to solve the following linear systems:

$$
\left(\begin{array}{cccc}
10 & -6 & 7.5 & -5 \\
-6 & 16 & -5 & -3 \\
-7.5 & 5 & 10 & -6 \\
5 & 3 & -6 & 16
\end{array}\right)\left(\begin{array}{l}
p_{1} \\
p_{2} \\
q_{1} \\
q_{2}
\end{array}\right)=\left(\begin{array}{c}
5 \\
-1 \\
0 \\
-2
\end{array}\right),
$$

$$
\left(\begin{array}{cccccccc}
10 & 0 & 7.5 & 0 & 0 & 6 & 0 & 5 \\
0 & 16 & 0 & 0 & 6 & 0 & 5 & 3 \\
0 & 5 & 10 & 0 & 7.5 & 0 & 0 & 6 \\
5 & 3 & 0 & 16 & 0 & 0 & 6 & 0 \\
0 & 6 & 0 & 5 & 10 & 0 & 7.5 & 0 \\
6 & 0 & 5 & 3 & 0 & 16 & 0 & 0 \\
7.5 & 0 & 0 & 6 & 0 & 5 & 10 & 0 \\
0 & 0 & 6 & 0 & 5 & 3 & 0 & 16
\end{array}\right)\left(\begin{array}{c}
p_{1}^{l} \\
p_{2}^{l} \\
q_{1}^{l} \\
q_{2}^{l} \\
p_{1}^{r} \\
p_{2}^{r} \\
q_{1}^{r} \\
q_{2}^{r}
\end{array}\right)
$$




$$
=\left(\begin{array}{l}
1 \\
1 \\
1 \\
1 \\
1 \\
1 \\
1 \\
1
\end{array}\right) .
$$

Form (29), the solution of the model is obtained as

$$
z=\left(\begin{array}{l}
p_{1} \\
p_{2} \\
q_{1} \\
q_{2}
\end{array}\right)=G^{\dagger}\left(\begin{array}{c}
5 \\
-1 \\
0 \\
-2
\end{array}\right)=\left(\begin{array}{c}
0.3542 \\
0.0654 \\
0.1086 \\
-0.2072
\end{array}\right),
$$

where

$$
G^{\dagger}=\left(\begin{array}{cccc}
0.0876 & 0.0389 & -0.0328 & 0.0224 \\
0.0389 & 0.0640 & 0.0224 & -0.0325 \\
0.0328 & -0.0224 & 0.0876 & 0.0389 \\
-0.0224 & -0.0325 & 0.0389 & 0.0640
\end{array}\right),
$$

$$
\left(\begin{array}{cc}
G^{+} & -G^{-} \\
-G^{-} & G^{+}
\end{array}\right)^{\dagger}=\left(\begin{array}{cccccccc}
0.1694 & -0.0015 & -0.0971 & 0.0061 & 0.0819 & -0.0404 & -0.0643 & -0.0612 \\
-0.0015 & 0.0725 & 0.0061 & 0.0171 & -0.0404 & 0.0085 & -0.0162 & -0.0154 \\
-0.0643 & -0.0162 & 0.1694 & -0.0015 & -0.0971 & 0.0061 & 0.0819 & -0.0404 \\
-0.0162 & -0.0154 & -0.0015 & 0.0725 & 0.0061 & 0.0171 & -0.0404 & 0.0085 \\
0.0819 & -0.0404 & -0.0643 & -0.0162 & 0.1694 & -0.0015 & -0.0971 & 0.0061 \\
-0.0404 & 0.0085 & -0.0162 & -0.0154 & -0.0015 & 0.0725 & 0.0061 & 0.0171 \\
-0.0971 & 0.0061 & 0.0819 & -0.0404 & -0.0643 & -0.0162 & 0.1694 & -0.0015 \\
-0.0061 & 0.0171 & -0.0404 & 0.0085 & -0.0162 & -0.0154 & -0.0015 & 0.0725
\end{array}\right)
$$

Since $p^{l}, q^{l}, p^{r}, q^{r}$ are nonnegative vectors, we obtain the fuzzy solution of the CFSLE as follows:

$$
\begin{aligned}
\widetilde{z} & =\left(\begin{array}{l}
\tilde{z}_{1} \\
\widetilde{z}_{2}
\end{array}\right) \\
& =\left(\begin{array}{c}
(0.3542,0.0378,0.0378)_{\mathrm{LR}}+i(0.1086,0.0378,0.0378)_{\mathrm{LR}} \\
(0.0645,0.0307,0.0307)_{\mathrm{LR}}+i(-0.2072,0.0307,0.0307)_{\mathrm{LR}}
\end{array}\right),
\end{aligned}
$$

and it admits a strong complex LR fuzzy solution.

Example 3. We consider an economic application of minimal solution of complex fuzzy linear systems. The market price of a good and the quantity produced are determined by the

$$
Y=\left(\begin{array}{c}
p_{1}^{l} \\
p_{2}^{l} \\
q_{1}^{l} \\
q_{2}^{l} \\
p_{1}^{r} \\
p_{2}^{r} \\
q_{1}^{r} \\
q_{2}^{r}
\end{array}\right)=\left(\begin{array}{cc}
G^{+} & -G^{-} \\
-G^{-} & G^{+}
\end{array}\right) \dagger\left(\begin{array}{c}
1 \\
1 \\
1 \\
1 \\
1 \\
1 \\
1 \\
1
\end{array}\right)
$$

$$
=\left(\begin{array}{l}
0.0378 \\
0.0307 \\
0.0378 \\
0.0307 \\
0.0378 \\
0.0307 \\
0.0378 \\
0.0307
\end{array}\right)
$$

where

equality between supply and demand. Suppose that demand and supply are linear functions of the price:

$$
\begin{aligned}
& q_{d}+a=b \times p+c \\
& q_{s}+d=e \times p+f
\end{aligned}
$$

where $q_{s}$ is the quantity supplied which is required to be equal to $q_{d}$, the quantity requested $p$ is the price, and $a, b, c, d, e$, and $f$ are coefficients to be estimated, where the coefficients $a, c, d$, and $f$ are represented by LR complex fuzzy numbers, and $b$ and $e$ are crisp numbers. By imposing the equality between quantity supplied and requested, 


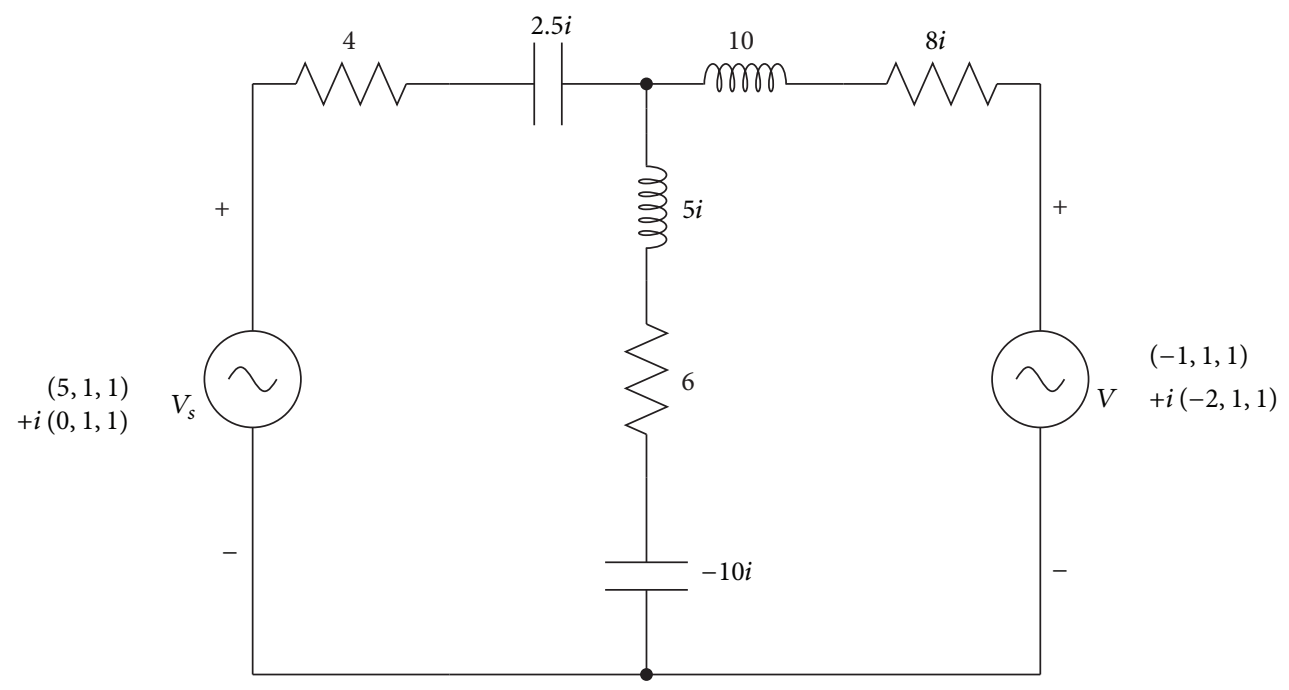

FIgURE 1: A RLC circuit with fuzzy current and fuzzy sources.

the following complex dual fuzzy linear system should be solved:

$$
\begin{aligned}
x_{1} & +((1,1,1)+i(2,1,1))_{\mathrm{LR}} \\
& =-\frac{1}{2} x_{2}+((3,2,2)+i(4,2,3))_{\mathrm{LR}}, \\
x_{1} & +((2,1,1)+i(1,2,1))_{\mathrm{LR}} \\
& =\frac{1}{2} x_{2}+((4,2,3)+i(5,3,3))_{\mathrm{LR}} .
\end{aligned}
$$

In fact, the above system is equivalent to a complex fuzzy linear system as follows:

$$
\left(\begin{array}{cc}
1 & \frac{1}{2} \\
1 & -\frac{1}{2}
\end{array}\right)\left(\begin{array}{l}
\tilde{x}_{1} \\
\tilde{x}_{2}
\end{array}\right)=\left(\begin{array}{l}
(2,1,1)_{\mathrm{LR}}+i(2,1,2)_{\mathrm{LR}} \\
(2,1,2)_{\mathrm{LR}}+i(4,1,2)_{\mathrm{LR}}
\end{array}\right) .
$$

Let $\widetilde{z}_{1}=\left(p_{1}, p_{1}^{l}, p_{1}^{r}\right)_{\mathrm{LR}}+i\left(q_{1}, q_{1}^{l}, q_{1}^{r}\right)_{\mathrm{LR}}, \widetilde{z}_{2}=\left(p_{2}, p_{2}^{l}\right.$, $\left.p_{2}^{r}\right)_{\mathrm{LR}}+i\left(q_{2}, q_{2}^{l}, q_{2}^{r}\right)_{\mathrm{LR}}$.

By using our proposed method, we need to solve the two following fuzzy systems:

$$
\begin{aligned}
& \left(\begin{array}{cc}
1 & \frac{1}{2} \\
1 & -\frac{1}{2}
\end{array}\right)\left(\begin{array}{l}
\widetilde{p}_{1} \\
\widetilde{p}_{2}
\end{array}\right)=\left(\begin{array}{l}
(2,1,1)_{\mathrm{LR}} \\
(2,1,2)_{\mathrm{LR}}
\end{array}\right), \\
& \left(\begin{array}{cc}
1 & \frac{1}{2} \\
1 & -\frac{1}{2}
\end{array}\right)\left(\begin{array}{l}
\widetilde{q}_{1} \\
\tilde{q}_{2}
\end{array}\right)=\left(\begin{array}{l}
(2,1,2)_{\mathrm{LR}} \\
(4,1,2)_{\mathrm{LR}}
\end{array}\right) .
\end{aligned}
$$

We can obtain easily via computation that

$$
(p, q)=\left(\begin{array}{cc}
1 & \frac{1}{2} \\
1 & -\frac{1}{2}
\end{array}\right)\left(\begin{array}{ll}
2 & 2 \\
2 & 4
\end{array}\right)=\left(\begin{array}{cc}
2 & 3 \\
0 & -2
\end{array}\right),
$$

$$
\begin{aligned}
\left(\left(\begin{array}{l}
p^{l} \\
q^{l}
\end{array}\right),\left(\begin{array}{l}
p^{r} \\
q^{r}
\end{array}\right)\right) & =\left(\begin{array}{rrrr}
1 & 0 & 0 & -\frac{1}{2} \\
1 & -\frac{1}{2} & 0 & 0 \\
0 & -\frac{1}{2} & 1 & 0 \\
0 & 0 & 1 & -\frac{1}{2}
\end{array}\right){ }^{\dagger}\left(\begin{array}{ll}
1 & 1 \\
1 & 2 \\
1 & 2 \\
1 & 2
\end{array}\right) \\
& =\left(\begin{array}{lll}
0.7500 & 1.2500 \\
1.0000 & 0.0000 \\
0.7000 & 1.7000 \\
0.6000 & 0.6000
\end{array}\right) .
\end{aligned}
$$

It means

$$
\begin{aligned}
& \widetilde{p}=\left(\begin{array}{l}
(2.0000,0.7500,0.2500)_{\mathrm{LR}} \\
(0.0000,1.0000,0.0000)_{\mathrm{LR}}
\end{array}\right), \\
& \widetilde{q}=\left(\begin{array}{c}
(3.0000,0.7000,1.7000)_{\mathrm{LR}} \\
(-2.0000,0.6000,0.6000)_{\mathrm{LR}}
\end{array}\right) .
\end{aligned}
$$

Since $\tilde{p}$ and $\tilde{q}$ are appropriate LR fuzzy numbers vectors, we obtained the solution of the complex fuzzy linear system which is

$$
\begin{aligned}
\widetilde{X}= & \tilde{p}+i \widetilde{q} \\
= & \left(\begin{array}{l}
(2.0000,0.7500,0.2500)_{\mathrm{LR}} \\
(0.0000,1.0000,0.0000)_{\mathrm{LR}}
\end{array}\right) \\
& +i\left(\begin{array}{c}
(3.0000,0.7000,1.7000)_{\mathrm{LR}} \\
(-2.0000,0.6000,0.6000)_{\mathrm{LR}}
\end{array}\right),
\end{aligned}
$$

and it admits a strong complex fuzzy solution by Definition 11. 


\section{Conclusion}

In this work we presented a matrix method for solving LR complex fuzzy linear equation $C \widetilde{z}=\widetilde{w}$, where $C$ is a crisp complex matrix and $\widetilde{w}$ is an arbitrary complex fuzzy vector. When a complex fuzzy numbers vector is expressed by this way, its operations for both computation and analysis will become more simple and easy. Numerical examples showed that our method is effective to solve the complex fuzzy linear system.

\section{Competing Interests}

The authors declare that there is no conflict of interests regarding the publication of this paper.

\section{Acknowledgments}

The work is supported by the Natural Scientific Funds of PR China (no. 61262022) and the Youth Scientific Research Promotion Project of Northwest Normal University (NWNULKQN-1120).

\section{References}

[1] L. A. Zadeh, "Fuzzy sets," Information and Control, vol. 8, no. 3, pp. 338-353, 1965.

[2] D. Dubois and H. Prade, "Operations on fuzzy numbers," International Journal of Systems Science, vol. 9, no. 6, pp. 613626,1978

[3] S. Nahmias, "Fuzzy variables," Fuzzy Sets and Systems, vol. 1, no. 2, pp. 97-110, 1978.

[4] M. L. Puri and D. A. Ralescu, "Differentials of fuzzy functions," Journal of Mathematical Analysis and Applications, vol. 91, no. 2, pp. 552-558, 1983.

[5] J. Goetschel and W. Voxman, "Elementary fuzzy calculus," Fuzzy Sets and Systems, vol. 18, no. 1, pp. 31-43, 1986.

[6] C. X. Wu and M. Ma, "Embedding problem of fuzzy number space: part I," Fuzzy Sets and Systems, vol. 44, no. 1, pp. 33-38, 1991.

[7] C. X. Wu and M. Ma, "Embedding problem of fuzzy number space. III," Fuzzy Sets and Systems, vol. 46, no. 2, pp. 281-286, 1992.

[8] M. Friedman, M. Ming, and A. Kandel, "Fuzzy linear systems," Fuzzy Sets and Systems, vol. 96, no. 2, pp. 201-209, 1998.

[9] S. Abbasbandy, R. Ezzati, and A. Jafarian, " $L U$ decomposition method for solving fuzzy system of linear equations," Applied Mathematics and Computation, vol. 172, no. 1, pp. 633-643, 2006.

[10] S. Abbasbandy, A. Jafarian, and R. Ezzati, "Conjugate gradient method for fuzzy symmetric positive definite system of linear equations," Applied Mathematics and Computation, vol. 171, no. 2, pp. 1184-1191, 2005.

[11] S. Abbasbandy, M. Otadi, and M. Mosleh, "Minimal solution of general dual fuzzy linear systems," Chaos, Solitons and Fractals, vol. 37, no. 4, pp. 1113-1124, 2008.

[12] T. Allahviranloo, "A comment on fuzzy linear systems," Fuzzy Sets and Systems, vol. 140, no. 3, pp. 559-560, 2003.
[13] T. Allahviranloo, "Numerical methods for fuzzy system of linear equations," Applied Mathematics and Computation, vol. 153, pp. 493-502, 2004.

[14] T. Allahviranloo, "Successive over relaxation iterative method for fuzzy system of linear equations," Applied Mathematics and Computation, vol. 162, no. 1, pp. 189-196, 2005.

[15] T. Allahviranloo, N. Mikaeilvand, and M. Barkhordary, "Fuzzy linear matrix equation," Fuzzy Optimization and Decision Making, vol. 8, no. 2, pp. 165-177, 2009.

[16] B. Asady, S. Abbasbandy, and M. Alavi, "Fuzzy general linear systems," Applied Mathematics and Computation, vol. 169, no. 1, pp. 34-40, 2005.

[17] M. Dehghan and B. Hashemi, "Iterative solution of fuzzy linear systems," Applied Mathematics and Computation, vol. 175, no. 1, pp. 645-674, 2006.

[18] M. Dehghan, B. Hashemi, and M. Ghatee, "Solution of the fully fuzzy linear systems using iterative techniques," Chaos, Solitons and Fractals, vol. 34, no. 2, pp. 316-336, 2007.

[19] K. Wang and B. Zheng, "Inconsistent fuzzy linear systems," Applied Mathematics and Computation, vol. 181, no. 2, pp. 973981, 2006.

[20] B. Zheng and K. Wang, "General fuzzy linear systems," Applied Mathematics and Computation, vol. 181, no. 2, pp. 1276-1286, 2006.

[21] T. Allahviranloo, M. Ghanbari, and R. Nuraei, "A note on 'Fuzzy linear systems,' Fuzzy Sets and Systems, vol. 177, no. 1, pp. 87-92, 2011.

[22] X. Guo and D. Shang, "Approximate solution of LR fuzzy Sylvester matrix equations," Journal of Applied Mathematics, vol. 2013, Article ID 752760, 10 pages, 2013.

[23] Z. T. Gong, X. B. Guo, and K. Liu, "Approximate solution of dual fuzzy matrix equations," Information Sciences, vol. 266, pp. 112133, 2014.

[24] J. J. Buckley, “Fuzzy complex numbers," Fuzzy Sets and Systems, vol. 33, no. 3, pp. 333-345, 1989.

[25] M. A. Jahantigh, S. Khezerloo, and M. Khezerloo, "Complex fuzzy linear systems," International Journal of Industrial Mathematics, vol. 2, pp. 21-28, 2010.

[26] T. Rahgooy, H. S. Yazdi, and R. Monsefi, "Fuzzy complex system of linear equations applied to ciccuit analysis," International Journal of Electrical and Computer Engineering, vol. 1, pp. 17938163, 2009.

[27] D. Behera and S. Chakraverty, "Solving fuzzy complex system of linear equations," Information Sciences, vol. 277, pp. 154-162, 2014.

[28] D. Behera and S. Chakraverty, "Erratum to 'Solving fuzzy complex system of linear equations', [Information Sciences, 277 (2014) 154-162]," Information Sciences, vol. 369, pp. 788-790, 2016.

[29] A. Ben-Israel and T. N. E. Greville, Generalized Inverses: Theory and Applications, Springer, New York, NY, USA, 2nd edition, 2003.

[30] R. J. Plemmons, “Regular nonnegative matrices," Proceedings of the American Mathematical Society, vol. 39, pp. 26-32, 1973.

[31] A. Berman and R. J. Plemmons, Nonnegative Matrices in the Mathematical Sciences, Academic Press, New York, NY, USA, 1979. 

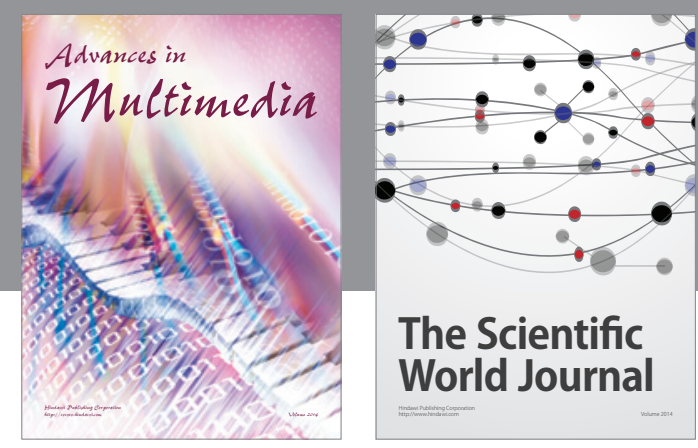

The Scientific World Journal
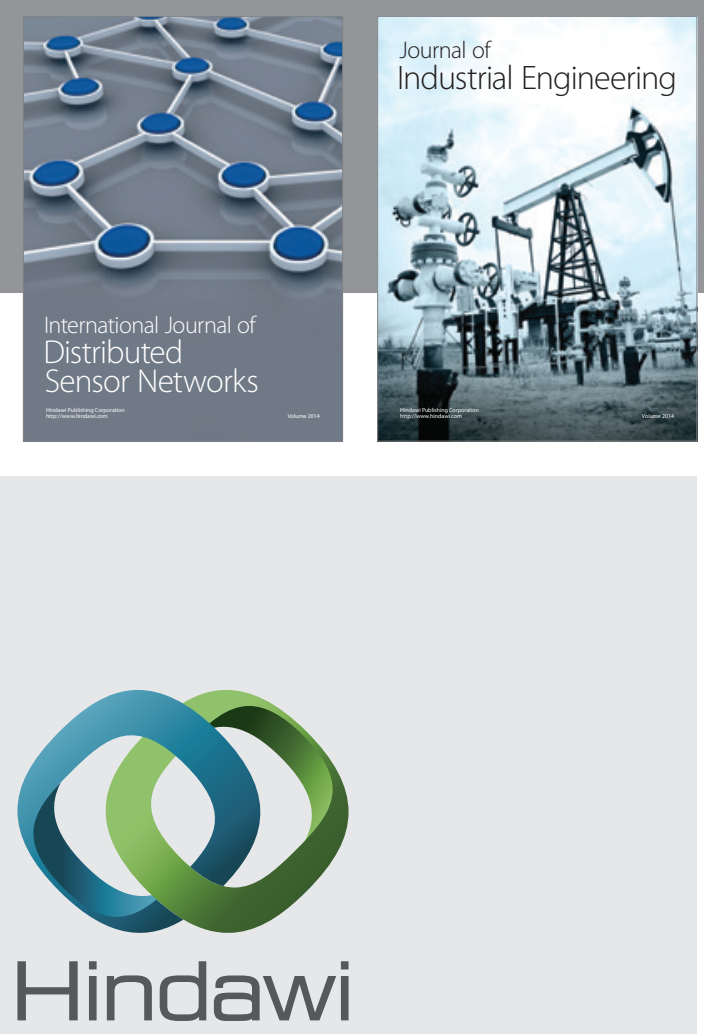

Submit your manuscripts at

http://www.hindawi.com

\section{Computer Networks} and Communications
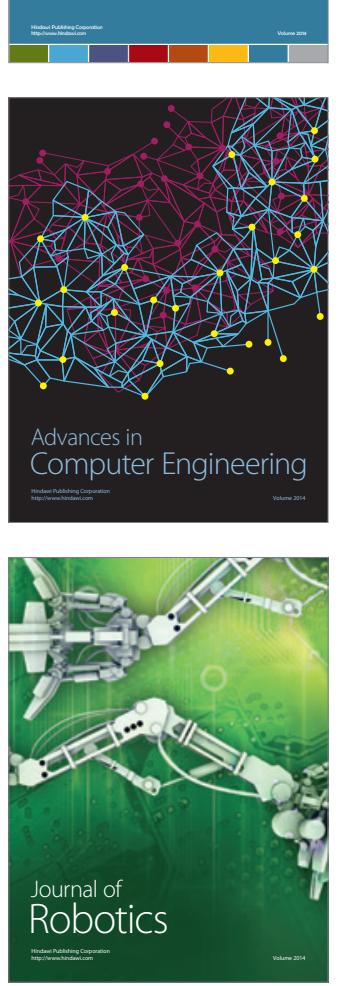
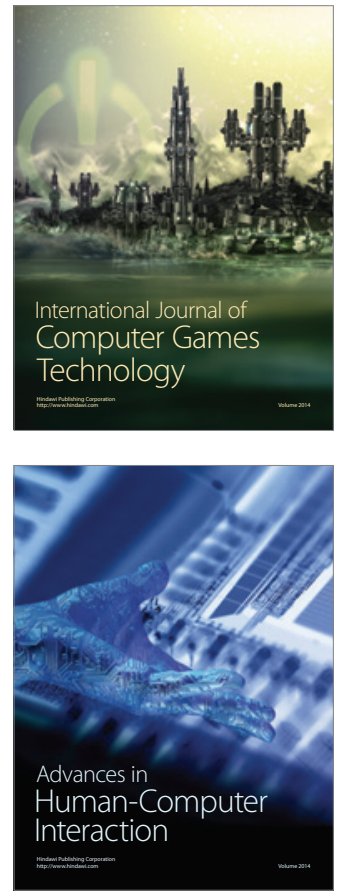
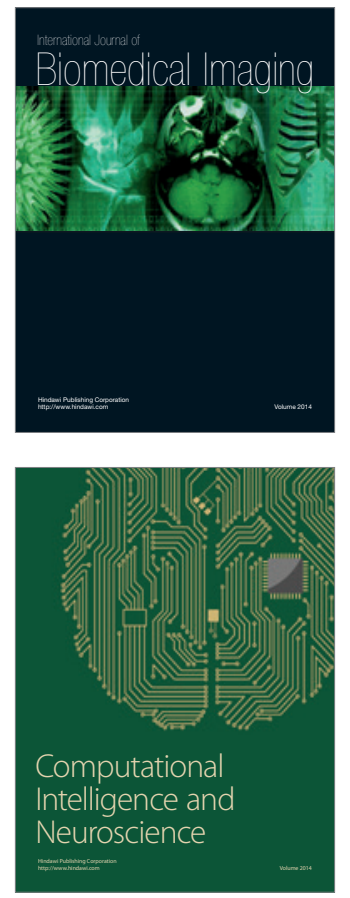
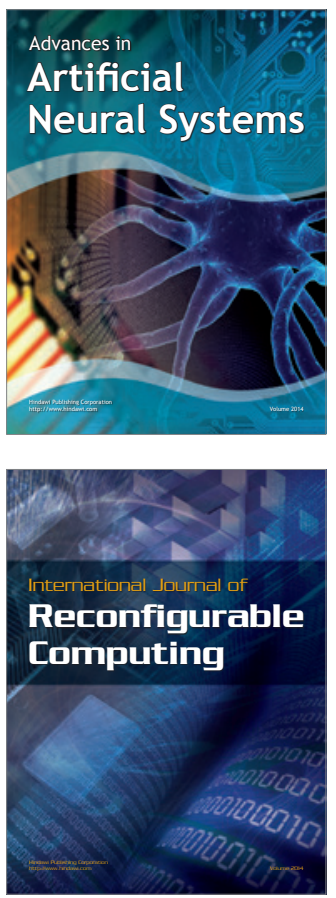
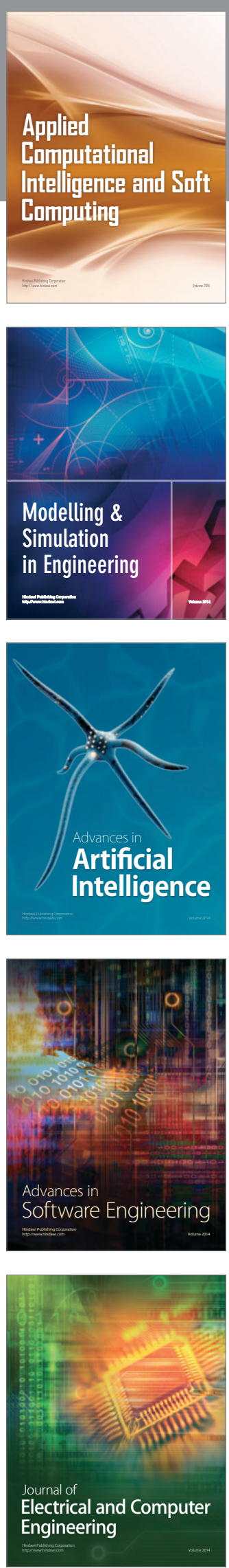